\title{
New Algorithms for Network Optimization
}

\author{
CHRISTAKIS CHARALAMBOUS, MEMBER, IEEE, AND JOHN W. BANDLER, MEMBER, IEEE
}

\begin{abstract}
Two new alogrithms suitable for computer-aided optimization of networks are presented. They are both based on the nonlinear least $p$ th approximation approach, which has been successfully applied by the authors to microwave network design problems requiring minimax or near-minimax solutions. A basic difference here is that, instead of requiring very large values of $p$, any finite value of $p$ greater than 1 can be used to produce extremely accurate minimax solutions. This paper discusses a six-vatiable transformer example where values of $p$ equal to $2,4,6,10,100,1000$, and 10000 have all been used separately to obtain substantially the same solution. Both the adjoint network method for gradient evaluation and the Fletcher method are employed for greater efficiency. Comparisons with the razor search and grazor search methods are made. Some far-reaching observations concerning minimax design are also made.
\end{abstract}

\section{INTRODUCTION}

I HE authors have already presented a justification of the use of least $p^{\text {th }}$ approximation techniques with large values of $p$ for computer-aided network design [1]. They showed that the use of a fairly well-conditioned objective function with efficient gradient minimization methods such as the method by Fletcher [2] and the adjoint network method for gradient evaluation [3], yields very near minimax designs with little computational effort.

The present paper exploits all the advantages of that approach in presenting two new algorithms for practical minimax approximation. A basic difference in these algorithms is that, instead of requiring very large values of $p$, any finite value of $p$ greater than 1 can be used to produce minimax solutions.

The paper discusses a six-variable example (namely, a three-section transmission-line transformer) where values of $p$ equal to $2,4,6,10,100,1000$, and 10000 have all been used separately to obtain substantially the same solution. A comparison with other methods already known to microwave engineers is made. The Fletcher minimization method is used throughout. The advantage of the new algorithms is a combination of efficiency and flexibility which, it is believed, has not been previously enjoyed by computer-aided circuit designers.

\section{The New Algorithms}

Most of the notation to be used here is the same as in a previous publication by the authors [1].

We denote by $F(\phi, \psi)$ the approximating function or network response, as indicated in Fig. 1. $\phi$ is a vector containing

Manuscript received May 16, 1973; revised August 13, 1973. This work was supported in part by the National Research Council of Canada under Grants $\mathrm{A} 7239$ and $\mathrm{C} 154$, and in part by a scholarship to $\mathrm{C}$. Charalambous. This paper was presented at the 1973 IEEE International Microwave Symposium, Boulder, Colo., June 4-6, 1973.

C. Charalambous was with the Department of Electrical Engineering McMaster University, Hamilton, Ont., Canada. He is now with the Department of Combinatorics and Optimization, University of Waterloo, Waterloo, Ont., Canada.

J. W. Bandler is with the Department of Electrical Engineering, McMaster University, Hamilton, Ont., Canada.

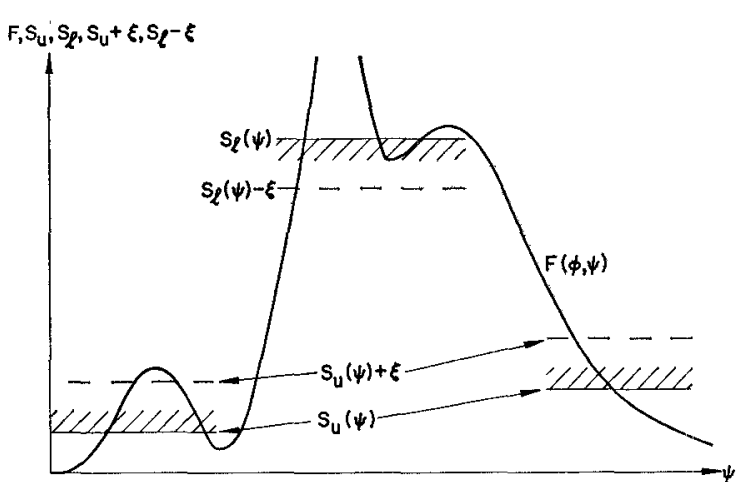

(a)
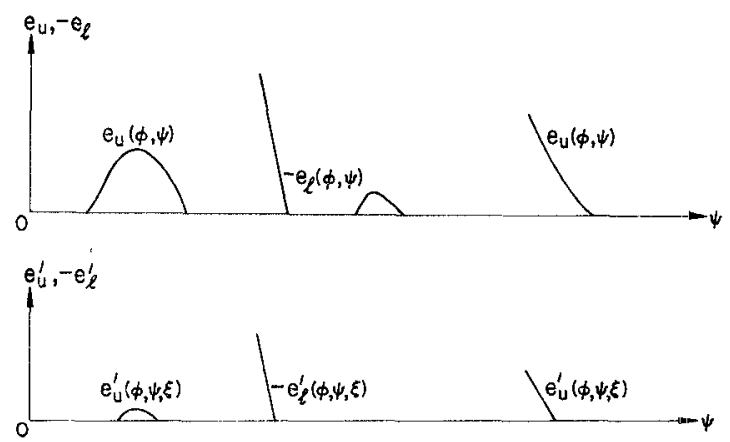

(b)

Fig. 1. An approximation problem where the response specifications are violated and the weighting factors are unity.

the $k$ independent variables, and $\psi$ is an independent variable, for example, frequency. $S_{u}(\psi)$ and $S_{l}(\psi)$ are upper and lower response specifications. $\xi$, as indicated in Fig. 1, is an artificial margin which plays an important role in the algorithms to be described. It is used to shift the error functions $e_{u}(\phi, \psi)$ $\triangleq w_{u}(\psi)\left(F(\boldsymbol{\phi}, \quad \psi)-S_{u}(\psi)\right)$ and $e_{l}(\boldsymbol{\phi}, \quad \psi) \triangleq w_{l}(\psi)(F(\boldsymbol{\phi}, \quad \psi)$ $\left.-S_{l}(\psi)\right)$ to obtain $e_{u}{ }^{\prime}(\boldsymbol{\phi}, \psi, \xi) \triangleq e_{u}(\boldsymbol{\phi}, \psi)-\xi$ and $e_{l}{ }^{\prime}(\phi, \psi, \xi)$ $\triangleq e_{l}(\boldsymbol{\phi}, \psi)+\xi$, respectively, where $w_{u}(\psi)$ and $w_{l}(\psi)$ are appropriate weighting functions.

As usual, we will sample the various functions to obtain, for example, $e_{u z}(\boldsymbol{\phi}) \triangleq e_{u}\left(\boldsymbol{\phi}, \psi_{i}\right)$ for $i \in I_{u}$ and $e_{i i}(\boldsymbol{\phi}) \triangleq e_{l}\left(\boldsymbol{\phi}, \psi_{i}\right)$ for $i \in I_{l}$, where the sets $I_{l b}$ and $I_{l}$ correspond to appropriate sample points. We also assume that these error functions are differentiable and that an optimum exists.

\section{Algorithm 1}

Step 1: $\xi^{1} \leftarrow \min \left[0, M_{e}\left(\dot{\phi}^{0}\right)+\epsilon\right]$, where $\boldsymbol{\phi}^{0}$ is the starting point, $\epsilon$ is a small positive number, and

$$
M_{e}(\boldsymbol{\phi}) \triangleq \max _{i, j}\left[e_{u i}(\boldsymbol{\phi}),-e_{l_{j}}(\boldsymbol{\phi})\right], \quad i \in I_{u}, j \in I_{l} .
$$

Step 2: $r \leftarrow 1$.

Step 3: Minimize with respect to $\phi$ the function 


$$
\begin{gathered}
U\left(\boldsymbol{\phi}, \xi^{r}\right) \leftarrow M\left(\boldsymbol{\phi}, \xi^{r}\right)\left(\sum_{i \in K_{i}}\left(\frac{e_{u i}(\boldsymbol{\phi})-\xi^{r}}{M\left(\boldsymbol{\phi}, \xi^{r}\right)}\right)^{q}\right. \\
\left.+\sum_{i \in K_{l}}\left(\frac{-e_{l i}(\boldsymbol{\phi})-\xi^{r}}{M\left(\boldsymbol{\phi}, \xi^{r}\right)}\right)^{q}\right)^{1 / q}, \quad \text { for } M\left(\boldsymbol{\phi}, \xi^{r}\right) \neq 0 \\
\quad U\left(\dot{\phi}, \xi^{r}\right) \leftarrow 0, \quad \text { for } M\left(\boldsymbol{\phi}, \xi^{r}\right)=0
\end{gathered}
$$

where

$$
\begin{gathered}
M\left(\boldsymbol{\phi}, \xi^{r}\right) \leftarrow M_{e}(\boldsymbol{\phi})-\xi^{r} \\
q \leftarrow p \operatorname{sign} M\left(\boldsymbol{\phi}, \xi^{r}\right)
\end{gathered}
$$

and

$$
\text { if } M(\phi, \xi)\left\{\begin{array}{c}
>0 \text { then } 1<p<\infty, K_{u} \leftarrow\left\{i \mid e_{u i}(\phi) \geq \xi^{r}, i \in I_{u}\right\} \\
\text { and } \left.K_{l^{\leftarrow}} \leftarrow i \mid-e_{l i}(\phi) \geq \xi^{r}, i \in I_{l}\right\} \\
<0 \text { then } 1 \leq p<\infty, K_{u} \leftarrow I_{u}, K_{l} \leftarrow I_{l} .
\end{array}\right.
$$

Step 4: $\xi^{r+1} \leftarrow M_{e}\left(\check{\phi}^{r}\right)+\epsilon$, where $\check{\phi}^{r}$ is the solution vector for Step 3.

Step 5: If $\left|\xi^{r+1}-\xi^{r}\right|<\eta$, stop. Otherwise $r \leftarrow r+1$. The quantity $\eta$ is a suitable small positive number.

Step 6: Go to Step 3.

Comments on Algorithm 1

If $M_{e}\left(\phi^{0}\right)$ is positive, $\xi^{1}=0$ and $q=p$, which is positive. If $M_{e}\left(\phi^{0}\right)$ is negative and for subsequent optimizations with $r=2,3, \cdots$, we have $q=-p$. We note that the reason for inclusion of $\epsilon$ in Steps 1 and 4 is to avoid having $M=0$. In this case, when two or more maxima in the error functions are equal, the objective function's first derivatives are discontinuous.

It can be shown [4] that the objective function decreases as $\xi$ increases, keeping the parameter vector $\boldsymbol{\phi}$ constant. As the sequence of optimizations proceeds for $r \geq 2$, the modulus of the objective function at each optimum in the sequence, namely $\left|U\left(\check{\boldsymbol{\phi}}^{r}, \xi r\right)\right|$, decreases as $r$ increases. Furthermore, as $r \rightarrow \infty,\left|U\left(\check{\boldsymbol{\phi}}^{r}, \xi\right)\right| \rightarrow 0$ and $M_{e}\left(\check{\boldsymbol{\phi}}^{r}\right) \rightarrow M_{e}(\check{\boldsymbol{\phi}})$, where $\check{\boldsymbol{\phi}}$ is the minimax solution vector desired [4].

\section{Algorithm 2}

Steps 1-3: As in Algorithm 1. wise

Step 4: If $M\left(\check{\phi}^{r}, \xi^{r}\right)<0$, remain with Algorithm 1. Other-

$$
\xi^{r+1} \leftarrow \xi^{r}+\lambda^{r} M\left(\check{\boldsymbol{\phi}}^{r}, \xi^{r}\right)=\left(1-\lambda^{r}\right) \xi^{r}+\lambda^{r} M_{e}\left(\check{\boldsymbol{\phi}}^{r}\right)
$$

where

$$
0<\lambda^{r}<1
$$

Steps 5 and 6: As in Algorithm 1.

\section{Comments on Algorithm 2}

Algorithm 2 differs from Algorithm 1 only when $M_{\epsilon}(\check{\phi})$ is positive. Under this condition $q=p$, which is positive. Furthermore, assuming we remain with Algorithm 2, the objective function at each optimum, namely, $U\left(\check{\phi}^{r}, \xi^{r}\right)$, decreases as $r$ increases. As $r \rightarrow \infty, U\left(\check{\boldsymbol{\phi}}^{r}, \xi r\right) \rightarrow 0$ and $M I_{e}\left(\check{\boldsymbol{\phi}}^{r}\right) \rightarrow M_{e}(\check{\boldsymbol{\phi}})$.

Algorithm 2 differs from Algorithm 1 in that Algorithm 2 attempts to predict $M_{e}(\check{\boldsymbol{\phi}})$ by increasing the level $\xi^{r}$, whereas Algorithm 1 attempts to push the maximum away from the
TABLE I

The Starting Points in the Optimization of a Three-Section 10:1 Transformer over 100-Percent Relative Bandwidth

\begin{tabular}{lcc}
\hline $\begin{array}{c}\text { Parameters } \\
\phi_{1}\end{array}$ & Problem 1 & Problem 2 \\
\hline$\ell_{1} / \ell_{q}$ \\
$z_{1}$ & 1.0 & 0.8 \\
$\ell_{2} / \ell_{q}$ & 1.0 & 1.5 \\
$z_{2}$ & 1.0 & 1.2 \\
$\ell_{3} / \ell_{q}$ & 3.16228 & 3.0 \\
$z_{3}$ & 1.0 & 0.8 \\
\hline $\begin{array}{l}\text { Maximum } \\
\text { reflection } \\
\text { coefficient }\end{array}$ & 10.0 & 6.0 \\
\hline
\end{tabular}

level $\xi$. To use Algorithm 2 when $M_{e}(\check{\phi})$ is negative, we simply add a suitably large positive constant value to all the error functions.

\section{EXAMPLES}

The algorithms have been applied to a wide range of design problems. Here, we will compare their performance for selected values of $p$ using the Fletcher method [2] on a threesection 100 -percent relative bandwidth $10: 1$ transmission-line transformer problem, which has already received attention in the literature [5], [6]. See Table I for a statement of the problem and the starting points used in the optimization. Specifically, we let $e_{u z}$ be the modulus of the reflection coefficient sampled at the 11 normalized frequencies

$$
\{0.5,0.6,0.7,0.77,0.9,1.0,1.1,1.23,1.3,1.4,1.5\} \text {. }
$$

Gradient vectors with respect to section lengths and characteristic impedances are obtained using the adjoint network method [3]. The number of function evaluations (one function evaluation comprising an evaluation of the objective function (2) and its derivatives) needed by Algorithms 1 and 2 to reach a reflection coefficient magnitude of 0.19729 , which is optimal to five figures, is shown in Tables II-IV. All six parameters were varied. Seven values of $p$ were tried ranging from 2 to 10000 . It is important to remember that the value of $p$ is fixed throughout each optimization so that the tables represent the effort required to reach substantially the same minimax solution for the particular value indicated.

Table II compares the present approach with previous results on the same problem. The paper by Bandler et al. [5] briefly discusses the method by Osborne and Watson [7]. The value of $\epsilon$ was $10^{-8}$.

Figs. 2 and 3 show how the maximum error $M_{e}$ defined in (1) is reduced at the end of each step in the sequence of optimizations.

As shown in Tables III and IV, different values of $\lambda$, the parameter given in (7), were used in testing Algorithm 2. In Table IV the quantity $M_{\theta}^{1}$ is the maximum error at the end of the first optimization for the particular value of $p$ indicated, and $m_{e}{ }^{1}$ is the corresponding value of the smallest ripple. On 
TABLE II

Optimization OF a Three-Section 10:1 Transformer OVer 100Percent Relative Bandwidth Using Algorithm 1

\begin{tabular}{lll}
\hline $\begin{array}{c}\text { Value } \\
\text { of } \\
\mathrm{p}\end{array}$ & $\begin{array}{c}\text { Number of function evaluations to reach a } \\
\text { reflection coefficient of } 0.19729\end{array}$ \\
\cline { 2 - 3 } & Problem 1 & Problem 2 \\
\hline 2 & 178 & 160 \\
4 & 143 & 128 \\
6 & 142 & 116 \\
10 & 112 & 89 \\
100 & 136 & 69 \\
1000 & 193 & 66 \\
10000 & 249 & 104 \\
\hline $\begin{array}{c}\text { Average number } \\
\text { of function } \\
\text { evaluations }\end{array}$ & 165 & 105 \\
\hline $\begin{array}{l}\text { Grazor } \\
\text { search [5] }\end{array}$ & 696 & 498 \\
\hline $\begin{array}{l}\text { Osborne } \\
\text { \& Watscn [5] }\end{array}$ & $860(0.20831)$ & $237(0.19788)$ \\
\hline $\begin{array}{l}\text { Razor } \\
\text { search [6] }\end{array}$ & $1300(0.19733)$ & $1250(0.19731)$ \\
\hline
\end{tabular}

a Number of function evaluations to reach the value shown in parentheses.

TABLE III

Optimization of a Three-Section 10:1 Transformer over 100Percent Relative Bandwidth Using Algorithm 2

\begin{tabular}{|c|c|c|c|c|c|c|}
\hline \multirow{3}{*}{$\begin{array}{c}\text { Value } \\
\text { of } \\
p\end{array}$} & \multicolumn{6}{|c|}{$\begin{array}{l}\text { Number of function evaluations to reach a } \\
\text { reflection coefficient of } 0.19729\end{array}$} \\
\hline & \multicolumn{2}{|c|}{ Problem 1} & \multirow[b]{2}{*}{$\lambda=0.7$} & \multicolumn{3}{|c|}{ Problem 2} \\
\hline & $\lambda=0.5$ & $\lambda=0.6$ & & $\lambda=0.5$ & $\lambda=0.6$ & $\lambda=0,7$ \\
\hline 2 & 183 & 146 & 151 & 165 & 128 & 133 \\
\hline 4 & 193 & 162 & 122 & 151 & 149 & 109 \\
\hline 6 & 199 & 182 & 138 & 150 & 135 & 112 \\
\hline 10 & 191 & 159 & 146 & 168 & 136 & 114 \\
\hline 100 & 185 & 171 & 165 & 126 & 104 & 99 \\
\hline 1000 & 211 & 211 & 202 & 83 & 91 & 83 \\
\hline 10000 & 248 & 248 & 248 & 103 & 103 & 103 \\
\hline $\begin{array}{l}\text { Average } \\
\text { number of } \\
\text { function } \\
\text { evaluations }\end{array}$ & 202 & 182 & 168 & 135 & 121 & 108 \\
\hline
\end{tabular}

the average, the choice of $\lambda$ shown in Table IV produced the best results. Note that $M_{e}{ }^{1}$ decreases as $p$ increases, as would be expected.

Figs. 4 and 5 illustrate ideas similar to those in Figs. 2 and 3. In addition, the rise of $\xi$ (relevant only in Algorithm 2) is also depicted.

\section{Discussion}

An important result of the investigation is that if the maximum error corresponding to the solution of the first optimization with any value of $p$ greater than 1 is positive (specification violated), then it remains positive for all per-
TABLE IV

Optimization OF a ThreE-Section 10:1 Transformer OVer 100Percent Relative Bandwidth Using Algorithm 2 with $\lambda=\left(M_{e}^{1}+m_{e}^{1}\right) / 2 M_{e}^{1}$

\begin{tabular}{|c|c|c|c|c|}
\hline \multirow[t]{2}{*}{$\begin{array}{l}\text { Value } \\
\text { of } \\
\mathrm{p}\end{array}$} & \multirow[t]{2}{*}{$M_{e}^{1}$} & \multirow[t]{2}{*}{$m_{e}^{1}$} & \multicolumn{2}{|c|}{$\begin{array}{l}\text { Number of function evaluations to } \\
\text { reach a reflection coefficient of } \\
0.19729\end{array}$} \\
\hline & & & Problem 1 & Problem 2 \\
\hline 2 & 0.30819 & 0.11626 & 161 & 141 \\
\hline 4 & 0.23680 & 0.16278 & 235 & 123 \\
\hline 6 & 0.21759 & 0.17612 & 115 & 89 \\
\hline 10 & 0.20636 & 0.18280 & 95 & 72 \\
\hline 100 & 0.19781 & 0.19562 & 131 & 65 \\
\hline 1000 & 0.19734 & 0.19712 & 193 & 66 \\
\hline 10000 & 0.19730 & 0.19727 & 249 & 104 \\
\hline \multicolumn{3}{|c|}{$\begin{array}{l}\text { Average number of function } \\
\text { evaluations }\end{array}$} & 155 & 95 \\
\hline
\end{tabular}

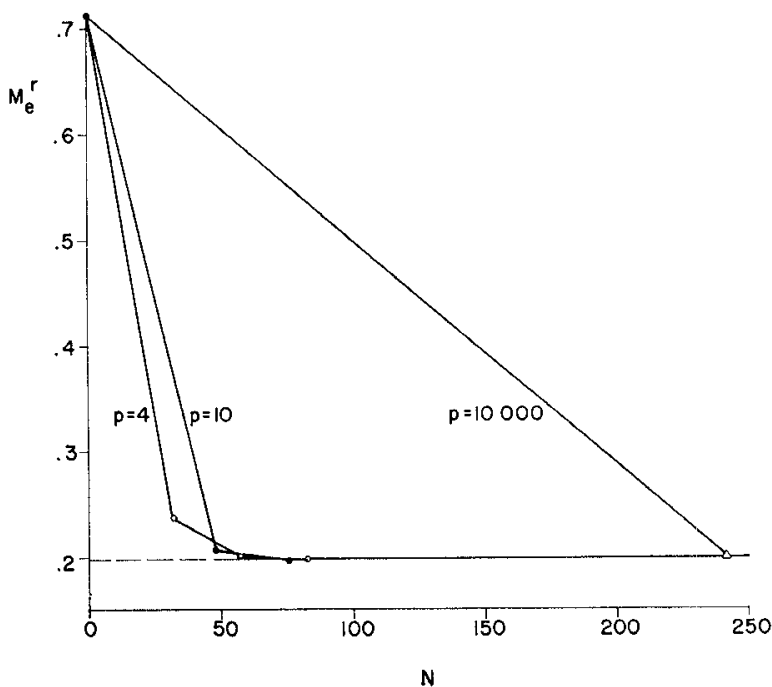

Fig. 2. Maximum error against number of function evaluations using Algorithm 1 on Problem 1.

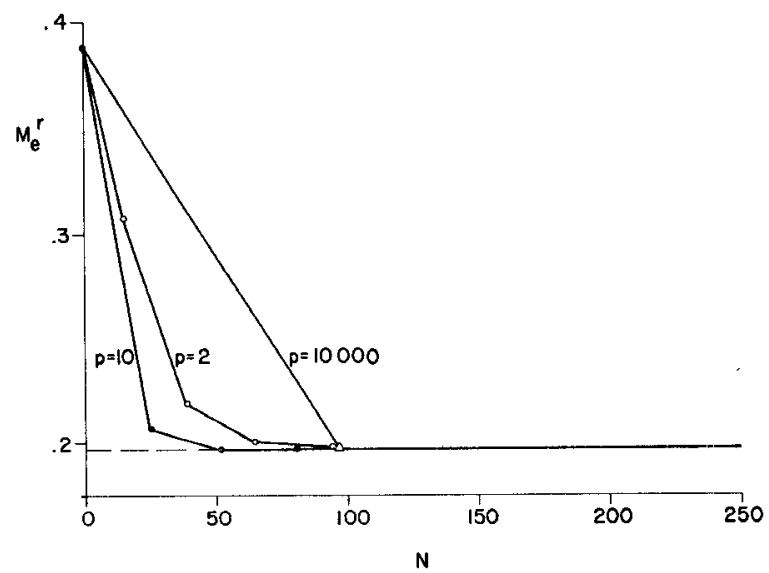

Fig. 3. Maximum error against number of function evaluations using Algorithm 1 on Problem 2. 


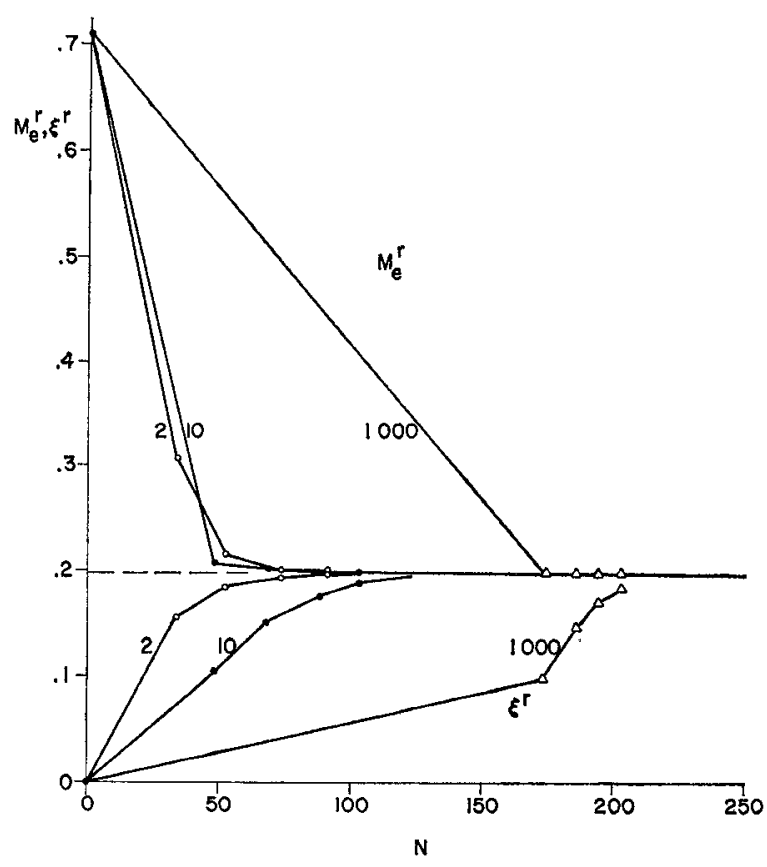

Fig. 4. Maximum error and $\xi$ against number of function evaluations using Algorithm 2 on Problem 1 with $\lambda=0.5$.

missible values of $p$. Similarly, if it is negative (specification satisfied), it remains negative for all permissible values of $p$. The same is true of the objective function in (2). Thus if we are investigating whether a particular structure will satisfy design specifications in the minimax sense, any single suitable least $p$ th optimization will reveal this! We do not need to carry out the full sequence of optimizations to obtain this information.

In practice, as the results indicate, only two or three optimizations with low values of $p$ will result in a good minimax design. For $p=10$, two optimizations usually suffice for practical purposes.

\section{Conclusions}

Two new algorithms for computer-aided minimax optimizations have been presented and discussed. Unlike previous work by the authors [1], where a single optimization is carried out with large $p$ in an effort to reach near minimax results, these algorithms can be described as true minimax algorithms. We believe that by adopting them, the designer will have an extremely flexible and efficient way of achieving minimax de-

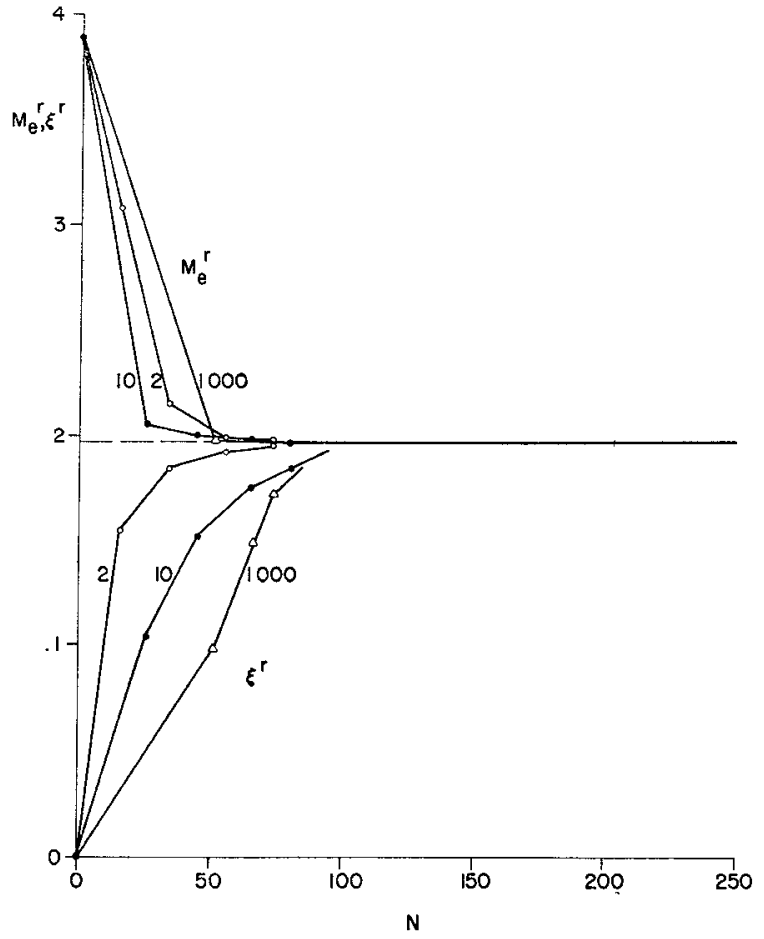

Fig. 5. Maximum error and $\xi$ against number of function evaluations using Algorithm 2 on Problem 2 with $\lambda=0.5$.

signs. The mathematical background with proofs of convergence, and so on, is rather involved and lengthy. It is available elsewhere [4].

\section{REFERENCES}

[1] J. W. Bandler and C. Charalambous, "Practical least pth optimization of networks," IEEE Trans. Microwave Theory Tech., vol. MTT20, pp. 834-840, Dec. 1972.

[2] R. Fletcher, "A new approach to variable metric algorithms," Comput. J., vol. 13, pp. 317-322, Aug. 1970.

[3] J. W. Bandler and R. E. Seviora, "Current trends in network optimization," IEEE Trans. Microwave Theory Tech., vol. MTT-18, pp. 1159-1170, Dec. 1970.

[4] C. Charalambous and J. W. Bandler, "Nonlinear minimax optimization as a sequence of least $p$ th optimization with finite values of $p$," McMaster Univ., Hamilton, Ont., Canada, Internal Rep. SOC-3 Simulation, Optimization, and Control, June 1973.

[5] J. W. Bandler, T. V. Srinivasan, and C. Charalambous, "Minimax optimization of networks by grazor search," IEEE Trans. Microwave Theory Tech., vol. MTT-20, pp. 596-604, Sept. 1972.

[6] J. W. Bandler and P. A. Macdonald, "Optimization of microwave networks by razor, search," IEEE Trans. Microwave Theory Tech. (Special Issue on Computer-Oriented Microwave Practices), vol. MTT17, pp. 552--562, Aug. 1969.

[7] M. R. Osborne and G. A. Watson, "An algorithm for minimax approximation in the non-linear case," Comput. $J$., vol. 12, pp. 63-68, Feb. 1969. 